\title{
Development of molecular and pharmacological switches for chimeric antigen receptor $T$ cells
}

\author{
Bill X. Wu ${ }^{*}$, No-Joon Song, Brian P. Riesenberg and Zihai Li
}

\begin{abstract}
The use of chimeric antigen receptor (CAR) T cell technology as a therapeutic strategy for the treatment blood-born human cancers has delivered outstanding clinical efficacy. However, this treatment modality can also be associated with serious adverse events in the form of cytokine release syndrome. While several avenues are being pursued to limit the off-target effects, it is critically important that any intervention strategy has minimal consequences on long term efficacy. A recent study published in Science Translational Medicine by Dr. Hudecek's group proved that dasatinib, a tyrosine kinase inhibitor, can serve as an on/off switch for CD19-CAR-T cells in preclinical models by limiting toxicities while maintaining therapeutic efficacy. In this editorial, we discuss the recent strategies for generating safer CAR-T cells, and also important questions surrounding the use of dasatinib for emergency intervention of CAR-T cell mediated cytokine release syndrome.
\end{abstract}

The validation of chimeric antigen receptor (CAR) $\mathrm{T}$ cell technology as a viable, therapeutic strategy for the treatment of blood-born human cancers has resulted in a renewed focus on ways to optimize and control this treatment modality $[1,2]$. CAR constructs are generated by linking an extracellular antibody-antigen recognition fragment with a costimulatory and intracellular activation domain from the $\mathrm{T}$ cell receptor complex, allowing for $\mathrm{T}$ cell recognition and clearance of tumors. The FDA has recently approved two CD19-directed CAR-T cell programs, Tisagenlecleucel (Kymriah) and Axicabtagene ciloleucel (Yescarta), for the treatment of various B cell malignancies including diffuse large $B$ cell lymphoma (DLBCL) and acute lymphoblastic leukemia (ALL). As is the case with other cancer treatments, CD19-CAR$\mathrm{T}$ cell therapy can also demonstrate serious, adverse side effects, the most common of which being cytokine release syndrome (CRS). CRS is characterized by massive and rapid releases of cytokines into the bloodstream, resulting in morbidity and even mortality in some

\footnotetext{
*Correspondence: xingjun.wu@osumc.edu; Zihai.li@osumc.edu Division of Medical Oncology, Pelotonia Institute for Immuno-Oncology, The Ohio State University Comprehensive Cancer Center, Columbus, OH
} 43210, USA patients. Different from other traditional cancer drugs, however, CAR-T cells are considered "living drugs" which may reside in patients for years, thereby increasing the probability for undesirable effects. Thus, development of precise techniques to manage the balance of activity and toxicity of CAR-T cells is of extreme importance.

Multiple strategies have been investigated experimentally, largely focusing on alterations to the CAR construct design, in order to generate safer CAR-T cell therapy (Table 1). Briefly, these strategies include introduction of suicide genes, dual targeted activation, inhibitory modules, and modification of structure to separate cytokine release from cytolytic signals. Incorporation of suicide genes, such as inducible caspase 9 (iCasp9) [3] and truncated epidermal growth factor receptor (EGFRt) [4], enables successful elimination of CAR-T cells in the event of abnormal activation by auxiliary treatment of patients with AP1903 or cetuximab, to activate iCasp9 or to target EGFRt respectively. Dual targeted activation strategy requires two tumor specific antigens recognized by CAR-T cells to be activated. This two-antigen recognition system helps CAR-T cells to avoid undesired activation by normal tissues while simultaneously aiding in anti-tumor activity and specificity of CAR-T cells [5].

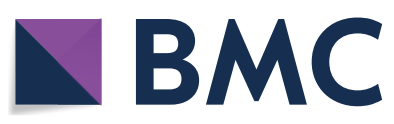

(c) The Author(s) 2019. This article is distributed under the terms of the Creative Commons Attribution 4.0 International License (http://creativecommons.org/licenses/by/4.0/), which permits unrestricted use, distribution, and reproduction in any medium, provided you give appropriate credit to the original author(s) and the source, provide a link to the Creative Commons license, and indicate if changes were made. The Creative Commons Public Domain Dedication waiver (http://creativecommons.org/ publicdomain/zero/1.0/) applies to the data made available in this article, unless otherwise stated. 
Table 1 Strategies for safer CAR-T therapy

\begin{tabular}{|c|c|}
\hline Strategy & Mechanism \\
\hline Suicide gene & Incorporation of suicide genes (iCasp9, EGFRt) $[3,4]$ \\
\hline Combinational targeted activation & Two antigen recognitions are required to fully activate CAR-T cells [5] \\
\hline Inhibitory CAR-T cells & Recognition of normal cell induces inhibition of CAR-T cell activity [6] \\
\hline CAR modification & $\begin{array}{l}\text { Modification of CAR molecules to identify constructs with less } \\
\text { cytokine production activity but with preserved anti-tumor func- } \\
\text { tion [7] }\end{array}$ \\
\hline On/off switch for CAR-T cell & Dasatinib serves as an on/off switch for CAR-T cells [8] \\
\hline
\end{tabular}
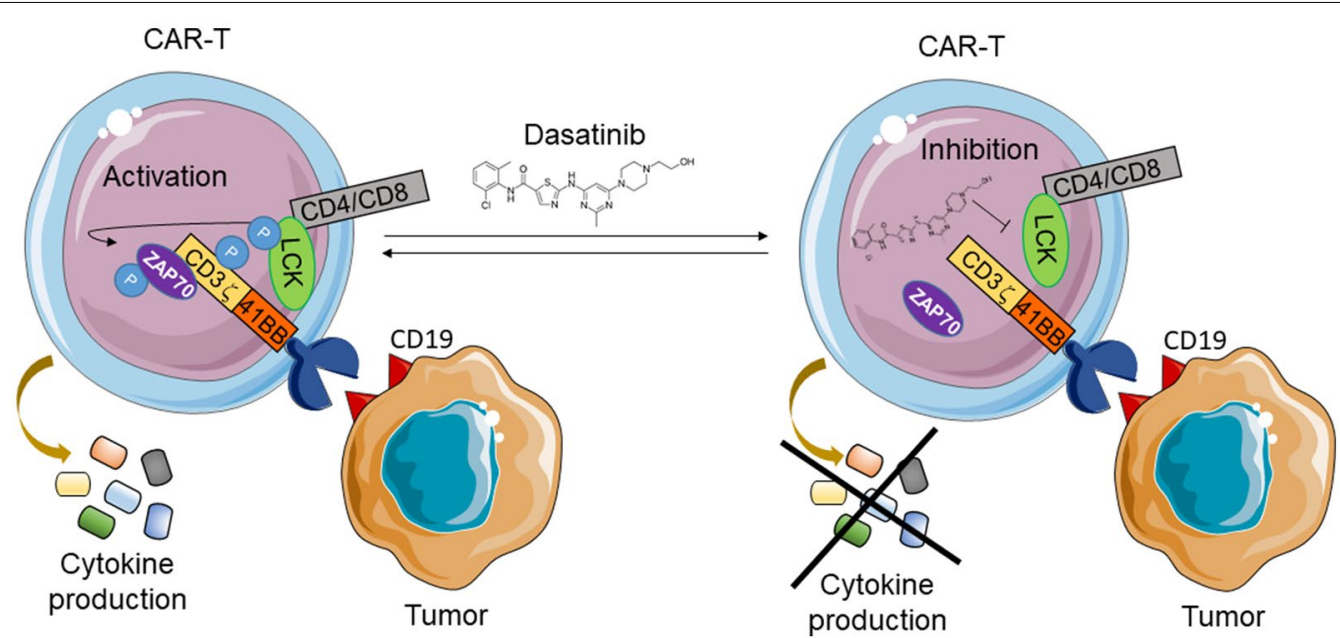

Fig. 1 Dasatinib, a tyrosine kinase inhibitor against BCR-ABL, can inhibit the phosphorylation and activation of components in the T cell receptor signaling pathways. It was shown to be effective in reversing CD19-CAR-T cell mediated cytokine release syndrome in a recently published preclinical model [8]

Similar to dual targeted activation, inhibitory CAR molecules contain an antigen recognition domain specific to the antigens expressed by normal tissues. Subsequent interaction between antigen and receptor in this setting arrests CAR-T cell activity [6]. Modifying the CAR construct has also been shown to provide safer treatment with CAR-T cells without diminishing clinical efficacy. Using a tertiary-structure-prediction program, Ying and colleagues were able to limit the toxicities associated with CAR-T cell therapy in a phase I clinical trial by altering the length of the transmembrane domain [7]. While these preliminary findings are exciting, uncovering a pharmacological intervention strategy allows for in vivo control of adverse toxicities while maintaining the anti-tumor response is an important endeavor.

In a recent study published in Science Translational Medicine, Mestermann et al. showed that dasatinib, a tyrosine-kinase inhibitor, mediated functional suppression of both $\mathrm{CD} 4^{+}$and $\mathrm{CD} 8^{+}$CD19-CAR-T cells in a dose-dependent manner [8] (Fig. 1). In vitro analysis with dasatinib demonstrated superior control over CAR-T cell function when compared to that of dexamethasone, an anti-inflammatory steroid used clinically to combat CAR-T cell toxicity [9]. From a mechanistic standpoint, dasatinib treatment abrogated phosphorylation of multiple key components in the CAR signaling domain, including lymphocyte specific protein tyrosine kinase (LCK), CD3 , and ZAP70. Importantly, the inhibitory effects were reversible as depletion of dasatinib immediately recovered CAR-T cell function and proliferation, both in vitro and in vivo, without compromising the long term efficacy of therapy. Finally, the authors validated dasatinib's potential as an emergency intervention strategy by using an acute CRS onset model [10]. In this setting, mice developed lethal CRS 2-3 days after CAR-T cell transfer. By initiating dasatinib $3 \mathrm{~h}$ after CAR-T transfer and continuing treatment for the next $30 \mathrm{~h}$, the authors were able to significantly enhance the immediate survival rate of mice. Taken together, the work presented by Mestermann et al. makes a convincing case for the potential use of dasatinib as an emergency drug to counter CAR-T mediated CRS in patients. 
As mentioned above, dasatinib is a tyrosine-kinase inhibitor which has been highly effective in treating patients with imatinib-resistant chronic myeloid leukemia (CML) and Philadelphia chromosome-positive ALL. However, as a nonspecific inhibitor, dasatinib has also been shown to interfere with other kinase family's function such as Src, Btk, and cKit to name a few [11]. Given the exciting work from Mestermann et al., several questions should be investigated prior to adopting the clinical use of dasatinib for controlling CAR-T cell toxicity. First, understanding how dasatinib influences CAR-T cell function in an immune competent setting should be examined. Current standard of care for CAR-T therapy requires a lymphodepletion regimen allowing for enhanced donor $T$ cell engraftment. Considering the complexity of immune reconstitution in this setting, it would be worthwhile to investigate the effects of dasatinib on this process. Secondly, how dasatinib treatment affects the endogenous tumor-specific $\mathrm{T}$ cell response should be addressed. The authors showed dasatinib had similar inhibitory effects when stimulating with an endogenous TCR-peptide combination in addition to stimulation with $\mathrm{CD} 19$, validating the broad inhibitory properties of the treatment. However, it is unclear if CAR-T cells are more sensitive to dasatinib compared to endogenous $\mathrm{T}$ cells due to increased antigen affinity as well as unique configuration of signaling cassette. Finally, it will be interesting to determine if other tyrosine kinase inhibitors have superior effects in inhibiting CAR-T cell mediated CRS by acting as a functional on/off switch.

The current paper from Dr. Hudecek's group proved that the tyrosine kinase inhibitor dasatinib can serve as an on/off switch for CD19-CAR-T cells in the preclinical models [8]. Clinical studies with dasatinib for the treatment of CRS induced by CAR-T therapy should be approached with caution until more can be elucidated surrounding the potential for off target effects. Nonetheless, the work presented by Mestermann and colleagues offers an enticing approach for reducing toxicity while maintaining efficacy of CAR-T cell therapy.

Acknowledgements

Not applicable.

Authors' contributions

BXW, NS, BPR and ZL contribute to writing the manuscript. All authors read and approved the final manuscript.
Funding

This work is supported by NIH Grants P01CA186866.

Availability of data and materials

Not applicable.

Ethics approval and consent to participate

Not applicable.

Consent for publication

Not applicable.

\section{Competing interests}

The authors declare that they have no competing interest.

Received: 8 September 2019 Accepted: 25 October 2019

Published online: 05 November 2019

\section{References}

1. Fesnak $A D$, June $C H$, Levine BL. Engineered T cells: the promise and challenges of cancer immunotherapy. Nat Rev Cancer. 2016;16:566-81.

2. June $\mathrm{CH}$, Sadelain M. Chimeric antigen receptor therapy. N Engl J Med. 2018;379:64-73.

3. Straathof KC, Pule MA, Yotnda P, Dotti G, Vanin EF, Brenner MK, Heslop HE, Spencer DM, Rooney CM. An inducible caspase 9 safety switch for T-cell therapy. Blood. 2005;105:4247-54.

4. Wang X, Chang WC, Wong CW, Colcher D, Sherman M, Ostberg JR, Forman SJ, Riddell SR, Jensen MC. A transgene-encoded cell surface polypeptide for selection, in vivo tracking, and ablation of engineered cells. Blood. 2011;118:1255-63.

5. Shah NN, Maatman T, Hari P, Johnson B. Multi targeted CAR-T cell therapies for B-Cell malignancies. Front Oncol. 2019;9:146.

6. Fedorov VD, Themeli M, Sadelain M. PD-1- and CTLA-4-based inhibitory chimeric antigen receptors (iCARs) divert off-target immunotherapy responses. Sci Transl Med. 2013:5:215ra172.

7. Ying Z, Huang XF, Xiang X, Liu Y, Kang X, Song Y, Guo X, Liu H, Ding N, Zhang T, Duan P, Lin Y, Zheng W, Wang X, Lin N, Tu M, Xie Y, Zhang C, Liu W, Deng L, Gao S, Ping L, Wang X, Zhou N, Zhang J, Wang Y, Lin S, Mamuti $M$, Yu X, Fang L, Wang S, Song H, Wang G, Jones L, Zhu J, Chen SY. A safe and potent anti-CD19 CART cell therapy. Nat Med. 2019;25:947-53.

8. Mestermann K, Giavridis T, Weber J, Rydzek J, Frenz S, Nerreter T, Mades A, Sadelain M, Einsele $H$, Hudecek M. The tyrosine kinase inhibitor dasatinib acts as a pharmacologic on/off switch for CART cells. Sci Transl Med. 2019;11:eaau5907.

9. Bonifant $\mathrm{CL}$, Jackson HJ, Brentjens RJ, Curran KJ. Toxicity and management in CAR T-cell therapy. Mol Ther Oncolytics. 2016:3:16011.

10. Giavridis T, van der Stegen SJC, Eyquem J, Hamieh M, Piersigilli A, Sadelain M. CART cell-induced cytokine release syndrome is mediated by macrophages and abated by IL-1 blockade. Nat Med. 2018;24:731-8.

11. Zarbock A. The shady side of dasatinib. Blood. 2012;1 19:4817-8.

\section{Publisher's Note}

Springer Nature remains neutral with regard to jurisdictional claims in published maps and institutional affiliations. 